\title{
Localização apical eletrônica em dentes decíduos - Revisão de lite- ratura
}

\author{
Amanda Caixeta de Araújo ${ }^{1}$, Carlos Rodolfo Mohn Neto². \\ ${ }^{1}$ Aluna de graduação no curso de Odontologia da Universidade Paulista - UNIP campus Goiânia; ${ }^{2}$ Professor Doutor da disciplina de Odon- \\ topediatria no curso de Odontologia da Universidade Paulista - UNIP campus Goiânia.
}

\begin{abstract}
Resumo
Objetivo: O objetivo do trabalho foi realizar uma revisão de literatura sobre a aplicabilidade dos localizadores apicais eletrônicos na determinação do comprimento de trabalho dos canais radiculares em dentes primários. Método: Esta pesquisa foi realizada a partir de consultas na literatura eletrônica, tanto em pacientes submetidos ao tratamento quanto em dentes extraídos. Foram encontrados artigos a partir da combinação dos descritores. Resultados: Os resultados evidenciaram aplicabilidade aceitável dos localizadores apicais eletrônicos de novas gerações, também em dentes decíduos, reduzindo a exposição a radiação, o tempo clínico e as limitações de radiografias. Conclusão: Conclui-se então, que localização apical eletrônica é um método auxiliar possível de ser aplicado durante a terapia endodôntica em dentes decíduos mesmo em alguns níveis de reabsorção radicular.
\end{abstract}

Palavras-chave: Dentes decíduos; Localizadores apicais eletrônicos; Pulpectomia em dentes primários; Comprimento do canal radicular.

\section{Introdução}

Os dentes primários, também "de leite" ou decíduos, ocupam a cavidade bucal de crianças por um período e depois são substituídos pelos dentes permanentes. Essa substituição não justifica perdas precoces, pois eles desempenham funções essenciais na mastigação e consequente digestão, manutenção de espaço e direcionamento de seus respectivos sucessores, desenvolvimento maxilofacial, de músculos e ossos da face, além de autoestima e fonação ${ }^{1,2}$

Os principais motivos de perda prematura de dentes primários são a cárie dentária, principalmente nos dentes posteriores por anatomia favorável a instalação e higiene menos eficaz, e traumatismos, nos dentes anteriores, levando a consequências que podem afetar também o sucessor permanen$t^{3,4}$. São fatores preventivos de cárie a utilização de fluoretos $^{5}$, a amamentação materna (mais protetora que mamadeiras) ${ }^{6}$, dieta e higiene, e quanto aos traumatismos e suas consequências, orientação de pais para monitorar e intervir corretamente.

Dependendo do comprometimento de um dente, indica-se alguma forma de terapia pulpar (capeamento pulpar, pulpotomia ou pulpectomia) ${ }^{7}$. O principal objetivo dentro da odontopediatria é manter os dentes primários até sua esfoliação fisiológica ${ }^{8}$, e as vezes para isso é necessário um tratamento, quando estes forem passíveis de reparação.

Na pulpectomia, é essencial a correta determinação do comprimento do canal para limpeza, instrumentação e obturação em níveis corretos ${ }^{9}$. O método radiográfico convencional é um dos mais utilizados para determiná-lo, mas apresenta algumas limitações, como: exposição aos raios $\mathrm{X}$, cooperação da criança, consultas longas, dificuldade na avaliação de pequenas reabsorções, imagem bidimensional de estrutura tridimensional, possibilidade de superposição e mane- jo da técnica pelo operador ${ }^{10,11}$. Para tentar superar tais limitações, iniciou-se estudos sobre a localização apical eletrônica, inclusive no tratamento de dentes decíduos.

Este trabalho tem como objetivo demonstrar, através de uma revisão de literatura, a aplicabilidade do método eletrônico para a localização apical na determinação do comprimento de trabalho em dentes decíduos.

\section{Método}

O presente artigo foi desenvolvido com base em estudos científicos publicados no período entre 1991 e 2017 em inglês e português nas bases de dados Scielo, Bireme e PubMed. Inicialmente, a busca foi feita utilizando-se os seguintes termos: "dentes decíduos", "localizadores apicais eletrônicos", "pulpectomia em dentes primários", "comprimento do canal radicular". A busca nas bases de dados resultou em 146 artigos e após a leitura e análise destes, foram selecionados 26 trabalhos que atenderam ao objetivo desta revisão de literatura: aplicabilidade dos localizadores apicais eletrônicos na determinação do comprimento de trabalho dos canais radiculares em dentes primários.

\section{Revisão da Literatura}

A anatomia dentária consiste na presença de coroa e raiz, composta por esmalte, dentina, cemento e polpa. Os dentes primários apresentam particularidades anatômicas, como esmalte e dentina menos espessos e polpa maior, com raízes mais divergentes, incompletas e com ápices abertos e canais radiculares mais amplos e cônicos, o que dificulta o tratamento endodôntico. Dependendo da gravidade e da possibilidade de manutenção de algum dente na cavidade bucal, o tratamento relacionado a polpa será pulpotomia ou pulpectomia. A pulpotomia consiste na remoção parcial da polpa dentária, aquela situada na por- 
ção coronária do dente, já a pulpectomia remove todo tecido infectado, tanto da coroa quanto da raiz, através de limpeza química e mecânica, e obturação, com um material que possa permitir a reabsorção normal ${ }^{1}$.

Para realização de pulpectomia, é essencial a determinação do comprimento para instrumentação e obturação em níveis corretos, evitando danos aos germes sucessores e aos tecidos adjacentes. Esse comprimento tem valores variáveis de 0 a $3 \mathrm{~mm}$ aquém do ápice radiográfico dependendo da presença ou não de bactérias, e pode ser medido de formas variadas ${ }^{12}$.

O método mais utilizado é a radiografia convencional através da técnica do paralelismo, porém apresenta algumas limitações, como: exposição à radiação, imagem bidimensional, técnica difícil pelo posicionamento do filme, processamento e armazenamento da radiografia, possibilidade de superposição de estruturas, maior demanda de tempo e cooperação, entre outros ${ }^{9}$.

$\mathrm{Na}$ tentativa de sobrepor as limitações das radiografias, surgiram os localizadores apicais eletrônicos, um avanço da tecnologia que são utilizados na determinação do comprimento de trabalho durante a terapia endodôntica, através de impedância elétrica, apresentando alta precisão, rapidez, conforto, não expõe à radiação, entre outros fatores que o destacam como método confiável e aplicável ${ }^{13}$. Os localizadores eletrônicos de primeira e segunda gerações não eram precisos em certos meios, como presença de irrigante, sangue, pus e tecidos pulpares, mas os mais recentes conseguem leitura fiel, mesmo com reabsorção ${ }^{14}$.

O método de determinação do comprimento do dente através do uso de localizadores apicais eletrônicos têm sido estudado e pesquisado quanto a aplicabilidade, também, em dentes decíduos. A utilização do localizador apical eletrônico Tri Auto ZX, comparado ao método radiográfico convencional, em dentes decíduos com e sem reabsorção de raízes, não apresenta diferença significativa e a presença de reabsorção não afeta a precisão do localizador ${ }^{11}$.

A medição de canais decíduos utilizando localizadores Root ZX II e Mini Apex em diferentes estágios de reabsorção pode ser realizada durante o tratamento endodôntico, pois a precisão pôde ser confirmada pela medição visual, em dentes uni ou multirradiculares e com ou sem reabsorção ${ }^{15}$. Tratamentos utilizando Root ZX II e sistema rotativo em dentes primários são feitos com maior rapidez, sem diferença significativa quando comparados com radiografias convencionais para determinação do comprimento do canal. Apresentam custo mais elevado, porém são considerados como coadjuvantes no tratamento endodôntico de dentes decíduos ${ }^{16}$.

A leitura do localizador apical eletrônico Root $Z X$ a $\pm 0,5 \mathrm{~mm}$ do forame apical apresenta precisão de $96,7 \%$, mesmo na presença de reabsorção radicular de molares primários ${ }^{17}$. Esse mesmo localizador, comparado a medição com métodos tátil, radiografia convencional, tátil + radiografia convencional e radiografia digital, apresenta-se o mais preciso, com $\pm 1 \mathrm{~mm}$ apical aceitável, e em segundo, método tá- til + radiografia convencional ${ }^{18}$. Comparando Root ZX ao método visual por observação direta e radiográfico pela técnica do paralelismo, conclui-se que pode ser um meio auxiliar no tratamento de molares decíduos $^{19}$, e também de dentes anteriores primários, com $86 \%$ de precisão do Root ZX e $76 \%$ da radiografia ${ }^{20}$.

A medição através de método eletrônico, utilizando localizador iPex multifrequência, mesmo na presença de reabsorção radicular fisiológica de dentes decíduos multirradiculares, é precisa de acordo com estudo ex vivo ${ }^{21}$. Também em estudo in vivo, esse localizador de ápice eletrônico de nova geração (iPex) tem sua precisão em dentes primários indicados para tratamento de pulpectomia, com ou sem reabsorção de raízes ${ }^{14}$.

O método de localização eletrônica, comparado ao uso de radiografias convencionais e digitais pela técnica de paralelismo, não apresenta diferença significativa, podendo ser utilizados com segurança na terapia endodôntica de dentes primários ${ }^{22}$. Os comprimentos de canal determinados por radiovisiografia e localizadores de ápice eletrônicos Propex II e Dentaport ZX são eficazes e auxiliares durante o tratamento necessário quando dentição decídua ${ }^{23}$.

\section{Discussão}

Um desafio na endodontia clínica é a localização da posição apical para determinar o comprimento de trabalho, o qual é determinado principalmente por meio radiográfico ou eletrônico. O limite ideal para o trabalho endodôntico é a junção cemento-dentinária, a qual nem sempre é coincidente com o ápice radiográfico ${ }^{24}$. O limite apical, além da junção cemento-dentinária, é ditado na zona de máxima contrição apical ou medição de 0,5 a $2 \mathrm{~mm}$ a partir da radiografia ${ }^{25}$.

A radiografia convencional é um método aceito, disponível e confiável para medição do comprimento do canal, porém possuem limitações de uso podendo sobrepor e distorcer imagens, necessitam posicionamento na boca, processamento e armazenamento de filme radiográfico, exposição a radiação, entre outros. Já os localizadores apicais eletrônicos, método eletrônico de medição dentária interna, são indolores, precisos, confiáveis, permitem rápida operação e alguns são capazes de detectar perfurações artificiais ${ }^{24}$. Os localizadores de primeira e segunda gerações geravam resultados imprecisos quando presença de polpa vital, exsudato, hemorragia e umidade excessiva, mas os aparelhos mais recentes e de novas gerações permitem medição precisa em várias circunstâncias pelo avanço tecnológico ${ }^{14}$.

A posição real do forame apical é, de certa forma, impossível de ser determinada radiograficamente, pela imagem pobre em detalhes e padrões de reabsorções assimétricas presentes nas raízes de dentes primários não visíveis na radiografia. Assim, a localização pode ser realizada através do método eletrônico buscando a redução de erros na mensuração, sobrepondo as limitações radiográficas ${ }^{16}$. Além disso, através do localizador apical eletrônico, é possível reduzir a exposição repetida 
aos raios $\mathrm{X}$, a qual é necessária antes, durante e depois do tratamento, além de acompanhamento periódico ${ }^{22}$. Quando há um forame aberto, como em dentes primários, a parede da dentina radicular é mais fina e consequentemente, pode afetar a impedância medida pelo localizador eletrônico. Com isso, uma leitura prematura pode ocorrer ${ }^{24}$. Mas, quando há presença de pequenas reabsorções radiculares, a visualização através de radiografias convencionais é limitada e nem sempre possível, podendo levar a instrumentação excessiva ou insuficiente ${ }^{11}$, com possibilidade de atingir 0 germe sucessor e/ou limitar o sucesso do tratamento.

Em casos em que há presença de discrepância entre os métodos de medição, deve-se dar preferência ao resultado obtido eletronicamente, desde que seja menor que $2 \mathrm{~mm}$ em comparação ao obtido por meio de radiografia. Na presença de discrepâncias maiores que $2 \mathrm{~mm}$, pode ser essencial radiografia adicional para avaliação de possível perfuração radicular ou mau funcionamento do aparelho localizador ${ }^{26}$.

Mesmo que a etapa da determinação do comprimento do dente, a partir de sua saída foraminal, possa ser realizada com um método mais rápido, seguro e não envolvendo radiações, através do meio eletrônico de localização, ainda é necessário radiografias para fornecimento de informações anatômicas e morfológicas da raiz ${ }^{17}$.

A literatura evidencia que a aplicabilidade dos localizadores apicais eletrônicos em dentes decíduos devia ser mais estudada ${ }^{11}$, mas após vários testes e pesquisas, uma meta-análise, no topo da evidência científica e dentro de suas limitações, concluiu que os localizadores apicais eletrônicos oferecem níveis aceitáveis na medida do comprimento de trabalho em dentes primários, mesmo em presença de reabsorções ${ }^{13}$.

\section{Considerações Finais}

Com base na literatura consultada, conclui-se que a localização apical eletrônica utilizada na endodontia de dentes decíduos é um método aplicável, seguro, capaz de reduzir a exposição do paciente a radiação ionizante, o tempo clínico, as limitações e dificuldades radiográficas. Seu uso como método auxiliar em dentes decíduos, protege o germe do sucessor permanente de instrumentação excessiva ou deficiente, com níveis de precisão aceitáveis.

\section{Referências}

1. Bolette A, Truong S, Guéders A, Geerts $S$. The importance of pulp therapy in deciduous teeth. Rev Med Liege, 2016; 71(12): 567-572.

2. Nogueira, A J S. Perdas precoces de dentes decíduos e suas conseqüências para dentição futura - elaboração de propostas preventivas. Rev ABO Nac, 1998; 6(4): 228-233.

3. Pinkham, J R. Odontopediatria da infância à adolescência. 2. ed. São Paulo: Artes Médicas, 1995; 393-415.
4. Holan G., Needleman, Howard L. Premature loss of primary anterior teeth due to trauma - potential short- and long-term sequelae. Dent Traumatol 2014; 30: 100-106.

5. Goodarzi F, Hossein AM, Hosseini M, Nabizadeh RN, Javad MK, Parvizishad M. Prevalence of dental caries and fluoride concentration of drinking water: A systematic review. Dent Res J, 2017; 14(3): 163-168.

6. Avila W. M, Pordeus I. A, Paiva S. M, Martins C. C. Breast and Bottle Feeding as Risk Factors for Dental Caries: A Systematic Review and Meta- Analysis. PLoS ONE, 2015; 10(11).

7. Smaïl-Faugeron V, Courson F, Durieux P, Muller-Bolla M, Glenny AM, Fron Chabouis H. Pulp treatment for extensive decay in primary teeth. Cochrane Database of Systematic Reviews 2014, Issue 8. Art. No.: CD003220.

8. American Academy of Pediatric Dentistry. Guideline on pulp therapy for primary and young permanent teeth. Pediat Dent, 2009; 31: 179-86.

9. Katz A, Tamse A, Kaufman AY, Aviv T. Tooth length determination: A review. Oral Surg Oral Med Oral Pathol, 1991; 72: 238-42. 10. Katz A, Mass E, Kaufman AY. Electronic apex locator: a useful tool for root canal treatment in the primary dentition. ASDC J Dent Child, 1996; 63: 414-7.

11. Mente J, Seidel J, Buchalla W, Koch MJ. Electronic determination of root canal length in primary teeth with andwithout root resorption. Int Endod J, 2002; 35: 447-452.

12. Wu MK, Wesselink PR, Walton RE. Apical terminus location of root canal treatment procedures. Oral Surg, Oral Med, Oral Pathol, Oral Radiol, and Endod, 2000; 89: 99-103.

13. Ahmad IA, Pani SC. Accuracy of electronic apex locators in primary teeth: a meta-analysis. International Endodontic Journal, 2014 [Acesso 08 maio 2018]. Disponível em: https://onlinelibrary. wiley.com/doi/abs/10.1111/iej.12315.

14. Bhat KV, Shetty $P$, Anandakrishna L. A Comparative Evaluation of Accuracy of New-generation Electronic Apex Locator with Conventional Radiography to determine Working Length in Primary Teeth: An in vivo Study. Int J Clin Pediatr Dent 2017; 10(1): 34-36.

15. Leonardo $M R$, Silva $L A B$, Nelson-Filho $P$, Silva RAB, Raffaini MSGG. Ex vivo evaluation of the accuracy of two electronic apex locators during root canal length determination in primary teeth. Inter Endod J, 2008; 41: 317-321.

16. Mokhtari N, Sarraf-Shirazi A, Ebrahimi M. A smart rotary technique versus conventional pulpectomy for primary teeth: $A$ randomized controlled clinical study. J Clin Exp Dent, 2017; 9(11): e1292-6.

17. Angwaravong O, Panitvisai P. Accuracy of an electronic apex locator in primary teeth with root resorption. Inter Endod J, 2009; 42: 115-121.

18. Mello-Moura ACV, Moura-Netto C, Araki AT, Guedes-Pinto AC, Mendes FM. Ex vivo performance of five methods for root canal length determination in primary anterior teeth. Inter Endod 
J, 2010; 43: 142-147.

19. Pinheiro SI, Bincelli In, Faria T, BUENO CES, CUNHA RS. Comparison between electronic and radiographic method for the determination of root canal length in primary teeth. RSBO, 2012; 9(1): 11-6.

20. Bahrololoomi Z, Soleymani AA, Modaresi J, Imanian M, Lotfian M. Accuracy of an Electronic Apex Locator for Working Length Determination in Primary Anterior Teeth. J Dent, 2015; 12(4): 243-248.

21. Nelson-Filho $P$, Romualdo $P C$, Bonifacio $K C$, Leonardo MR, Silva RAB, Silva LAB. Accuracy of the iPex multifrequency electronic apex locator in primary molars: an ex vivo

study. Inter Endod J, 2011; 44: 303-306.

22. Neena IE, Ananthraj A, Praveen P, Karthik V, Rani P. Comparison of digital radiography and apex locator with the conventional method in root length determination of primary teeth. J Ind Soc Of Pedod Prev Dent, 2011; 29(4): 300-4.

23. Abdullah A, Singh N, Rathore MS, Tandon S, Rajkumar B. Comparative Evaluation of Electronic Apex Locators and Radiovisiography for Working Length Determination in Primary Teeth in vivo. Int J Clin Pediatr Dent 2016; 9(2): 118-123.

24. Kim E, Lee S-J. Electronic apex locator. Dent Clin North Am 2004; 48(1): 35-54.

25. Ricucci D. Apical limit of root canal instrumentation and obturation. Part 1. Literature review. Int Endod J, 1998; 31: 384-93.

26. Elayouti A. The ability of Root ZX apex locator to reduce the frequency of overestimated radiographic working length. J Endod, 2002; 28(2): 116-119.

\section{Autor Correspondente:}

Prof. Dr. Carlos Rodolfo Mohn Neto* Rodovia BR 153, KM 503, Fazenda Botafogo, Goiânia/ GO, CEP 74.845-090.

Telefone: +55 (62) 3239-4000

E-mail: carl.mohn@hotmail.com

Recebido em : 31/08/2018

Aprovado em: 29/11/2018

Os autores declaram que não há conflitos de interesse.

\title{
Electronic apical location in deciduous teeth - Review of literature
}

\begin{abstract}
Aim: The objective of this work was to perform a literature review on the applicability of electronic apical locators in determining the working length of root canals in primary teeth. Methods: This research was carried out from consultations in the electronic literature, both in patients submitted to treatment and in extracted teeth. Articles were found from the combination of descriptors. Results: The results showed acceptable applicability of the electronic apical locators of new generations, also in deciduous teeth, reducing exposure to radiation, clinical time and radiographic limitations. Conclusion: It is concluded that apical electron location is a possible auxiliary method to be applied during endodontic therapy in deciduous teeth even at some levels of root resorption.
\end{abstract}

Keywords: Deciduous teeth; Electronic apical locators; Pulpectomy in primary teeth; Root canal length. 